\title{
The ST3 Mission, and its Capabilities for Be Star Observations
}

\author{
R. P. Linfield \\ Infrared Processing and Analysis Center, California Institute of \\ Technology, Pasadena, CA 91125
}

\begin{abstract}
The ST3 mission will launch a space optical interferometer into heliocentric orbit, for observation of 50-100 sources on baselines of 40-200 m, during a time span of 3-4.5 months. The angular resolution in the $450-900 \mathrm{~nm}$ passband will be $\approx 500$ microarcseconds. For Be stars, a velocity resolution of $50-100 \mathrm{~km} / \mathrm{s}$ can be achieved by operating the interferometer in a Fourier Transform Spectrometer mode. This should allow spectrally resolved measurements of the brightness distribution in circumstellar disks. but will be limited to stars brighter than a visual magnitude of $\approx 5$.
\end{abstract}

\section{Introduction}

The New Millenium Program of the National Aeronautics and Space Administration (NASA) was formed to test and demonstrate new technologies for use in future space missions. The Space Technology Three (ST3) mission, scheduled for launch in 2005, will place two spacecraft into heliocentric orbit to do optical interferometry in the $450-900 \mathrm{~nm}$ band (the original mission concept involved three spacecraft, but budget limitations forced a descope of the mission and its capabilities). Each spacecraft will have a $12 \mathrm{~cm}$ diameter siderostat to capture starlight. Key technologies to be demonstrated by ST3 include: autonomous formation flying with multiple spacecraft, delay and delay rate estimation using angular laser and starlight metrology, and Michelson interferometry in space using multiple free flying spacecraft. The autonomous formation flying system, using GPS-like radio frequency transmitters and receivers, will allow the range between spacecraft to be controlled to a few centimeters, and the relative angles (spacecraft attitudes and the orientation of the vector between the two spacecraft) to be controlled to a few arcminutes.

With a fixed delay line on one spacecraft (the 'combiner'), zero total delay can be achieved by placing the second spacecraft on the surface of a parabaloid, whose axis points towards the target star. For a fixed delay line of length $\tau_{\text {fixed, }}$, the relation between the $y$-coordinate (along the axis of the parabaloid) and the projected baseline length $B$ is:

$$
y=\frac{B^{2}}{2 \tau_{\text {fixed }}}-\frac{\tau_{\text {fixed }}}{2}
$$

With a $20 \mathrm{~m}$ fixed delay line, and a maximum allowed range between spacecraft of $1000 \mathrm{~m}$, ST3 can achieve projected baselines up to $200 \mathrm{~m}$ in length. The min- 
imum projected baseline length will be $40 \mathrm{~m}$-the two spacecraft must remain $\geq 50 \mathrm{~m}$ apart for safety reasons.

Operating above the earth's atmosphere allows a big improvement in sensitivity over ground-based optical interferometers. Rapid atmospheric delay fluctuations limit the coherence time of a ground interferometer to a few milliseconds. For a space interferometer, almost all the power in delay fluctuations will occur at frequencies $<<1 \mathrm{~Hz}$. For ST3, we expect that low power thruster firings ( $\sim 100 \mu \mathrm{N} \mathrm{s}$ ), at intervals of several hundred to several thousand seconds, plus a small, constant acceleration from solar radiation pressure, will be the only significant disturbances to the spacecraft motions. As a result, the coherence time will be $>>1 \mathrm{~s}$. Once fringes are detected, coherent integration for many seconds can be used to achieve a high signal-to-noise ratio, even on weak sources.

The challenge is to detect fringes in the first place, and this process will be limited primarily by the uncertainty in the interferometer delay rate. With one or two guide interferometers (such as with SIM), the delay rate will be accurately known, but ST3 will not have this capability. The delay rate knowledge will be derived from a time series of angle measurements, using both laser and starlight metrology.

This method of delay rate estimation will be applicable to the Terrestrial Planet Finder (TPF) mission. In its astrophysics mode (as opposed to planet search mode), many targets will be so weak that a guide interferometer will be needed. The brightest guide star in a typical field of view will be 18th magnitude at the $2.2 \mu \mathrm{m}$ wavelength used for fringe search and tracking. Frinding fringes with TPF will require delay rate knowledge 4-5 times better than is needed for the ST3 design point $\left(V=8\right.$ and $\left.\Gamma_{\text {star }}=0.3\right)$. Because the maximum TPF baseline will be $\approx 1000 \mathrm{~m}$ (five times longer than for ST3), the required angle rate sensing accuracy will be $\sim 25$ times more stringent for TPF than for ST3.

\section{Science Capabilities of ST3}

Aperture synthesis with ST3 will involve moving the two spacecraft, in order to change the vector baseline. During the planned science phase of 3-4.5 months, 50-100 sources can be observed. For many sources, only one-dimensional $u$ - $v$ coverage will be obtained, in order to limit the use of time and spacecraft fuel. More detailed two-dimensional coverage will be obtained for a limited subset of sources.

The fringe phase will be measured during ST3 observations. However, the uncertainty in the geometric delay $(0.2-2 \mathrm{~cm})$ will result in thousands of cycles of uncertainty in the geometric component of the fringe phase. The structure (visibility) phase will therefore be completely unknown, and only the visibility amplitude can be determined, in general. ST3 will be restricted to simple source structure measurements, generally derived from model fitting instead of a true inversion of the visibility data.

The expected ('design point') detection limit for ST3 will be a visual magnitude $V=8$ for sources with visibility $\Gamma_{\text {star }}=0.3$. For $\Gamma_{\text {star }}=0.7$ (adequate for measuring the angular sizes of simple sources), the limit will be 1.5 magnitudes fainter. For $M$ stars, where most of the flux is at wavelengths longer than in the $\mathrm{V}$ band, the detection limit (in $\mathrm{V}$ ) will be 1.5 magnitudes fainter than for hotter 
(B through $\mathrm{G}$ ) stars. Thus an $\mathrm{M}$ star as faint as $V=11$ may be detectable for $\Gamma_{\text {star }}=0.7$.

\section{Science Targets of Interest}

The science capabilities of ST3 (40-200 m baselines, detection limit of $V=8$ 10 ), are comparable to those anticipated for several ground-based interferometers (e.g. NPOI, CHARA) at the time of ST3's launch. ST3 is primarily a technology demonstration mission, and is not driven by science requirements. However, we have identified three types of objects for which ST3 observations may yield interesting new science, beyond that possible with current ground-based interferometers.

\subsection{Be stars}

The key observational challenge for Be star observations with ST3 will be to measure the structure of the circumstellar material that is producing the emission lines (it may be possible to measure the size and oblateness of the stellar photosphere for a few of the very brightest Be stars). The widths of Be-star emission lines are a few hundred $\mathrm{km} / \mathrm{s}$, much narrower than the $\sim 4000 \mathrm{~km} / \mathrm{s}$ width of a CCD spectral channel on ST3. Furthermore, a spectral resolution several times finer than the total Be star line width is desired, in order to study the dynamics of the circumstellar material. Therefore, the standard observing mode (tracking the fringes with half the white light, and dispersing the other half for measurement) cannot be used. We plan to operate ST3 in a Fourier Transform Spectrometer mode for Be star observations.

In this mode, fringe-searching will be done in the same way as for other stars. Once fringes are found, the delay line will be swept back and forth, over a range as large as 2000-3000 $\mu \mathrm{m}$. The detected signal (amplitude and phase) vs. delay, in each CCD spectral channel, will be Fourier Transformed to yield the correlated flux (and phase) vs. wavelength. This can be divided by the total spectral intensity (from ground-based photometry) to derive the complex visibility. The TPF project hopes to include this observing mode in their mission; a successful demonstration by ST3 would be valuable. We expect that stars as faint as $V \approx 5$ can observed by ST3 with a velocity resolution of $\approx 70 \mathrm{~km} / \mathrm{s}$. Unlike ground-based observations which use a filter to obtain visibility measurements in a single narrow spectral band, ST3 observations will measure the narrow-band visibility simultaneously across the full $450-900 \mathrm{~nm}$ band.

ST3 measurements should be able to address some of the following issues on Be stars. Simple measurements of the two-dimensional brightness distribution of the circumstellar disks should yield their axial ratio (inclination) and position angle of the disk. Measurements of the angular size in a few spectral lines should allow the radial density distribution to be estimated (or at least constrained). Phase referencing of the emission line visibilities to the continuum (photosphere) visibility will yield information on azimuthal brightness variations (e.g. onearmed spirals). A first order fit for the velocity field should be possible, allowing a test for Keplerian vs. angular momentum conserving vs. co-rotating disks. 
Finally, by tracing the visibility function out past its first minimum, it will be possible to test for the presence of a gap in the center of the circumstellar disk.

There are constraints on the pointing direction of ST3, due to the dual requirements of sufficient sunlight on the solar panels (for spacecraft power) and shielding of the optical components from sunlight. There are two main consequences for science observations. First, two-dimensional $u$ - $v$ coverage cannot be obtained at a single epoch. Instead, observations at two epochs $\sim 3$ months apart must be combined. As a result, study of time-variable structure will be difficult. Second, full two-dimensional coverage can only be obtained for sources that are more than $\sim 40^{\circ}$ from the ecliptic.

\subsection{Wolf-Rayet stars}

Wolf-Rayet stars (Abbott \& Conti 1987) are hot (effective temperature $\sim 30,000 \mathrm{~K})$, with very strong outflows $\left(10^{-5}-10^{-4} \mathrm{M}_{\odot} / \mathrm{yr}\right)$. In the continuum, ST3 will see the stellar surface, which will be compact and unresolved. This continuum emission can be used for the detection and tracking of fringes. A few of the strongest emission lines have widths comparable to the ST3 CCD channel width of $1 / 80$ octave $(\sim 4000 \mathrm{~km} / \mathrm{s})$, so that the measured visibility in that CCD channel will reflect the visibility from the region producing the emission line.

As for Be stars, the compact continuum can serve as a phase reference, allowing measurements of both the visibility amplitude and phase in the strong emission lines. As a generalization for simple outflow shapes, the visibility amplitude can be used to derive the radial density profile and aspect ratio (flattening) of the outflow. The visibility phase will be sensitive to any asymmetry (i.e. whether the outflow is stronger on one side of the star than the other).

\subsection{Dwarfs}

The effective temperature scale of the lower main sequence is poorly known. The spectra of $\mathrm{M}$ stars deviate strongly from a blackbody curve, so the connection between spectra and effective temperature is not simple. Current ground-based interferometers can measure the angular sizes of $M$ giants and supergiants, but not $M$ dwarfs.

ST3 measurements of the angular sizes to an accuracy of $2.5 \%$ would allow effective temperature determinations to $\approx 5 \%$, a factor of $\sim 4$ better than current knowledge. For visibilities in the range $0.5-0.7$, a visibility calibration accuracy of $\Delta \Gamma_{\mathrm{star}} \approx 0.02$ is needed to achieve a size accuracy of $2.5 \%$. The sensitivity of ST3 should allow M dwarfs as cool as $\approx \mathrm{M} 6$ to be observed.

The web site for ST3 is http://spacetechnology3.jpl.nasa.gov

\section{References}

Abbott, D. C., \& Conti, P. S. 1987, ARA\&A, 25, 113

Note added June 2000: Due to budget problems, the ST3 mission has been forced to significantly reduce its capabilities. As a result, useful interferometric measurements of Be-stars will not be possible. We still plan to demonstrate the technology of separated spacecraft interferometry, in order to pave the way for future, more capable missions. 